\title{
Parametric Influences on Heat Affected Zone in Micro-channel Milling Process of Zirconia Ceramic
}

\author{
Omar Faruk Biswas ${ }^{1 *}$, Abhishek Sen², Ishwer Shivakoti , Golam Kibria \\ ${ }^{1}$ Department of Mechanical Engineering, Aliah University, Action Area II-A/27, Newtown, 700156 Kolkata, West Bengal, India \\ 2 Department of Mechanical Engineering, Calcutta Institute of Technology, 6 National Highway, Banitabla, Uluberia, \\ 711316 Howrah, West Bengal, India \\ ${ }^{3}$ Department of Mechanical Engineering, Sikkim Manipal Institute of Technology, Sikkim Manipal University, 737136 Majitar, \\ Sikkim, India \\ * Corresponding author, e-mail: faruk.au91@gmail.com
}

Received: 16 November 2020, Accepted: 27 December 2020, Published online: 17 March 2021

\begin{abstract}
Zirconia, a bio-ceramic, is widely utilized in bioengineering, biomedical implants, dentistry, and the automotive industry due to high hardness, excellent wear resistance, etc. However, it is difficult to attain micro features on zirconia utilizing a laser machining system for the aforesaid properties. The paper deals with the study of the Heat Affected Zone (HAZ) formation during the micro-channel milling (V-shaped cross-sections) of zirconia utilizing a nanosecond fiber laser system. Experiments are accomplished to examine the consequence of control variables namely transverse feed, pulse frequency, laser power, scan number, and scan speed. The influence of each of the laser process variables on response parameter are studied in order to get the significant trends of laser parameters. With the increment of laser power, the number of pass and transverse feed, HAZ width dimensions tend to increase. The reverse phenomena are observed for pulse frequency and scan speed. The lower HAZ width which is achieved as $31.74 \mu \mathrm{m}$ at a parametric setting of average power at $10 \mathrm{~W}$, pulse frequency at $65 \mathrm{kHz}$, scanning speed at $11 \mathrm{~mm} / \mathrm{s}$, a number of pass at 1 , transverse feed at $0.005 \mathrm{~mm}$.
\end{abstract}

Keywords

fiber laser, micro-channel, micro-milling, HAZ, zirconia

\section{Introduction}

In the last two decades, micro-machining using a laser beam has showcased enormous potential during the machining of advanced engineering materials like polymer, ceramics, composites, etc. with the aid of different machining procedures for the generation of micro-parts in present manufacturing industries. The field of laser micro-machining procedures includes various manufacturing methods like micro-cutting, micro-turning, micro-drilling, micro-grooving, spiral micro-grooving, welding, and material surface texturing of materials irrespective of the material properties $[1,2]$. Predominantly, to remove material from the workpiece surface with the aid of laser system, three material removal mechanisms are involved, i.e., ejection of melting materials, vaporization, and further direct ablation. Microchannel milling with the help of a laser beam is one of the newly evolved techniques having different applications in bioengineering, aerospace, automotive, microelectronics, cryogenic systems, thermal and power industries $[3,4]$.
Ceramic materials have exceptional mechanical properties which include high-temperature withstand, high melting point, low coefficient of friction, extreme hardness, etc. It is very difficult to machine these types of material by conventional machining processes due to its brittleness properties and surface damage initiated during machining $[5,6]$. Laser beam machining is very suitable for machining these types of hard-to-machine material. When laser beam irradiates on workpiece surface of the ceramics material then the physical phenomena which taking place are reflection, absorption, scattering and transmission. Zirconia ceramic has gained a lot of attention in biomedical field due to high fracture toughness, corrosion resistance, lower thermal conductivity, electrical insulation, modulus of elasticity similar to steel which make the material to be utilized in different field including engineering, medical mainly for orthopedic and dental application, aerospace, etc. [7]. 
Various analytical and experimental investigations are performed by the investigator to study the influences of several process variables on Heat Affected Zone formation around the micro-channel milling zone. A study was conducted on laser milling of the CFRP sheet (4 mm thick) by utilizing a Yb: YAG fiber laser of $30 \mathrm{~W}$ [8]. The authors considered pulse frequency, scanning speed, repetitions of the geometric pattern, scanning strategy, and gap between two successive scan lines. They concluded that HAZ increases due to heat diffusion (at a lower speed or higher frequency) as well as the buildup of the affected zone of work material. Zhao et al. [9] proposed a technique of real-time adaptive control of laser fluence according to actual feed rate. The researchers developed a predictive tool to obtain the desired depth of milling. Experimental results showed that using the conventional constant laser fluence will lead to inhomogeneous milling depth and excessive HAZ. Darwish et al. [10] carried out studies on micro-channel milling on Ti alloy by neodymium-doped YAG laser. The authors deduced that the dimension of the manufactured channel expands with laser intensity and pulse frequency. Besides, they found that the micro-channel opening is decreasing with the scan speed. Under dissimilar setting of laser variables, the desired micro-channel of $100 \mu \mathrm{m}$ was achieved. The least micro-channel spatter dispersion was obtained at settings of the pulse repetition rate of $35 \mathrm{kHz}$, a scan speed of $400 \mathrm{~mm} / \mathrm{s}$ as well as lamp current intensity of $82 \%$. Wang et al. [11] performed laser induced plasma micro-machining utilizing a 10 ps laser to generate micro-channels on the work sample surface of $\mathrm{Al}_{2} \mathrm{O}_{3} / \mathrm{TiC}$ ceramic. The researchers investigated the effect of laser process parameters such pulse energy, frequency and scanning speed on machining characteristics during the process. They found that Heat Affected Zone width increased with the increment of overlap and pulse energy. Another group of researchers manufactured textured grooves using laser (pulsed) ablation (wavelength $1064 \mathrm{~nm}$ ) on zirconia ceramics [12]. The authors presented the study of process variables on HAZ in the region of the groove. They obtained groove size as $30-50 \mu \mathrm{m}$ in width and $15-50 \mu \mathrm{m}$ in depth. It was concluded that laser process variables have a significant effect on HAZ.

It is observed from the literature survey that micro-channels are one of the significant characteristics but quality of the micro-channels i.e. geometry, surface roughness, Heat Affected Zone etc. are vital issue for the application purposes. Nanosecond laser provides better quality surface as compared to millisecond laser due to shorter pulse duration. However, nanosecond and picosecond laser offers almost similar outcomes in terms of machining rate while machining of zirconia ceramics by laser beam [13, 14]. Dear et al. [15] found that nanosecond laser was better alternative for flexibility as well as precision machining of zirconia ceramics. Though few experimentations are performed on zirconia for the machining of micro-channel and the effect of laser variables on the HAZ width of micro-channel has not been examined. In this study an attempt has been accomplished for micro-machining of zirconia by fiber laser beam machining operating with $1064 \mu \mathrm{m}$ wavelength. The influences of each of the laser process parameters (laser power, scan speed, pulse frequency, number of pass and transverse speed) are studied to analyze the effect on HAZ width. The main aim of the research work is to showcase a cost-effective method to generate micro-channel on zirconia for the utilization in dental implants and dental restoration. The current study thus provides an extensive study of laser micro-channel milling zirconia.

\section{Materials and methods}

In this work, zirconia is taken as a workpiece material with dimensions (width of $25 \mathrm{~mm}$, breadth of $25 \mathrm{~mm}$, thickness $5 \mathrm{~mm}$ ) for the generation of micro-channels by utilizing multi-diode pumped fiber laser (50 W power). Ytterbium acts as the gain media. The diagramed view of the pulsed fiber laser system is depicted in Fig. 1.

The fiber laser beam machining system includes various subsystems such as laser beam generation unit, CCD camera, CCTV, $X-Y-Z$ controller, compressed air supply unit computer for controlling laser power. Besides, the detailed specification of the multi diode pump fiber laser set-up is shown in Table 1.

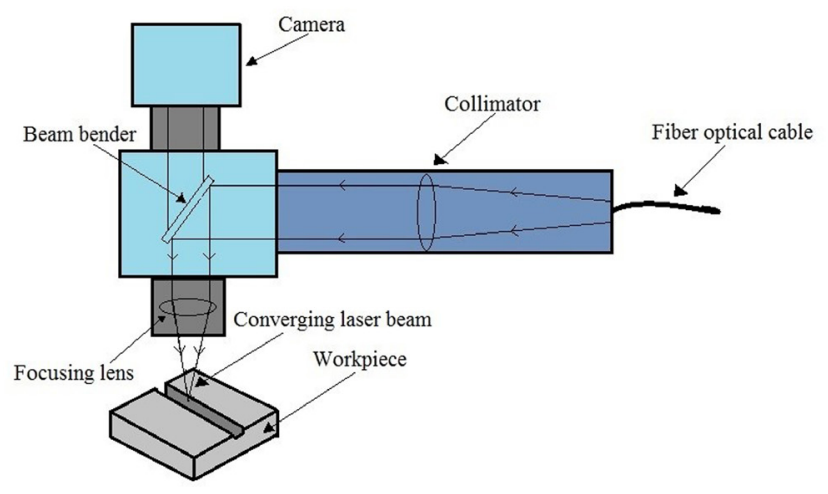

Fig. 1 Schematic diagram of multi diode pumped pulsed fiber laser system 
Table 1 Detail specifications of fiber laser set-up.

\begin{tabular}{lc}
\hline Specifications & Descriptions: \\
\hline Laser type & Multi Diode Pump Fiber Laser \\
Mode of operation & Pulsed \\
Wavelength & $1064 \mathrm{~nm}$ \\
Pulse Repetition Rate & $50-120 \mathrm{kHz}$ \\
Pulse duration & $120 \mathrm{~ns}$ \\
Beam diameter at collimator & $9 \mathrm{~mm}$ \\
Nominal Average Power & $50 \mathrm{~W}$ \\
Max. peak power & $7.5 \mathrm{KW}$ (approx.) \\
\hline
\end{tabular}

The experimentation was executed in pulsed mode. It causes high peak power, which is enabling generating high energy during machining. A high amount of energy can cause high surface roughness, excessive HAZ, etc. on the workpiece surface, which is not desirable for the micro-channel profile. Table 2 shows the various properties and their values of zirconia ceramic. The spot diameter of the laser beam is measured as $43 \mu \mathrm{m}$.

In the present research, the laser beam interacts with the square-shaped workpiece, which is progressed in the $X$-axis direction for scanning followed by the $Y$-axis direction. In this process, material is removed layer-bylayer from flat shaped workpiece surface to generate micro-channel on difficult-to-machine materials.

In the laser micro-channel milling, various phenomena follow on the micro-milling zone, e.g., heating, melting, evaporation, ejection, etc. Initially, the concentrated laser beam is focused onto the workpiece surface and the spot with higher energy density is created. The thermal energy accrued on the substrate surface further increases the temperature of the machining zone. The workpiece material starts decomposing into various particles. Further, melted due to the absorption of laser energy. The energy distribution of the laser beam is similar to the Gaussian profile. Thus, the absorption of energy is higher at the center as compared to the edges of the micro-channel. The surface beside the micro-channel may be affected due to the uneven distribution of heat as well as shock waves on the surface $[16,17]$. Because of this fact, Heat Affected Zone

Table 2 Properties of zirconia ceramics

\begin{tabular}{lc}
\hline Properties & Values \\
\hline Density & $5.68 \mathrm{~g} / \mathrm{cm}^{3}$ \\
Melting point & $2,715^{\circ} \mathrm{C}$ \\
Young's modulus & $210 \mathrm{GPa}$ \\
Compressive strength & $2000 \mathrm{MPa}$ \\
Bend strength & $900-1200 \mathrm{MPa}$ \\
Hardness & $1200 \mathrm{HV}$ \\
\hline
\end{tabular}

(HAZ) beside the micro-channel is generated in which material is not melted but microstructure and mechanical properties are affected. This may cause various effects that are not desirable like cracking, distortion, fatigue resistance, etc. of surfaces. Therefore, it is a challenging task for the researcher to select the proper process parameters and generation of micro-channel with lower HAZ [18, 19]. In this study, the Heat Affected Zone on the width of the micro-channel is examined. A schematic diagram of the formation of the micro-channel milling process on the workpiece surface is shown in Fig. 2. The micro-channels are fabricated by the reciprocating motion of the laser beam along the scanning area and the material is removed in a layer by layer. It shows that ejected/molten material, which is removed from the surface during machining and recast layer is also formed from the resolidified molten material at the side walls. Fig. 3 shows the schematic representation of the generation of micro-channels.

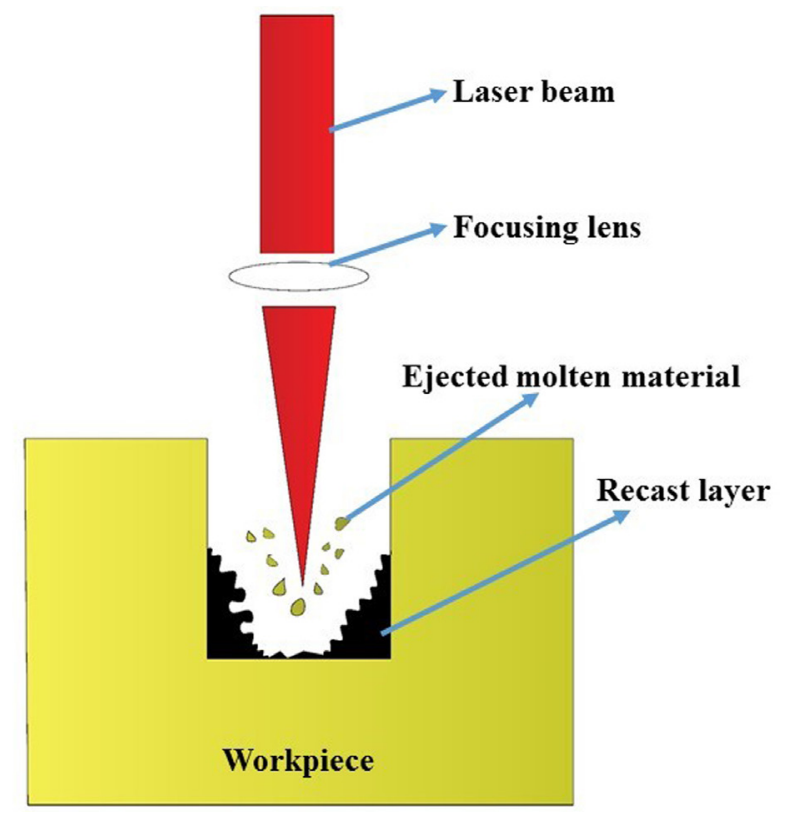

Fig. 2 A schematic diagram of the formation of micro-channels

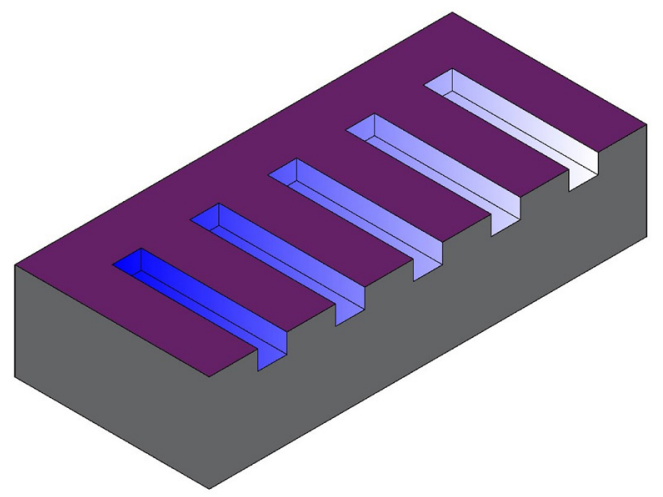

Fig. 3 Schematic representation of micro-channel 
It is seen during the experimentation that there are two aspects which are intricate for the quality fabrication of micro-channel are transverse overlap and spot overlap. Details of the laser scanning approach, including spot and transverse overlap, are shown in Fig. 4.

Equation (1) [20] depicts the mathematical relationship of the spot overlap and various process variables.

Spot Overlap Percentage $=\left(1-\frac{v}{D \times f}\right) \times 100 \%$

Scan speed is denoted by $v(\mathrm{~mm} / \mathrm{s})$; spot diameter is denoted by $D(\mathrm{~mm})$; pulse frequency is denoted by $f(\mathrm{~Hz})$. From Eq. (1), it is obvious that the amount of spot overlap depends on the scanning speed $(v)$ and pulse frequency $(f)$ of the laser beam. The value of the percentage of spot overlap increases with the increase of pulse frequency at constant laser spot diameter and scanning speed. This is because of the fact that, as the pulse frequency increases the number of laser beam strikes on the workpiece surface also increases which causes the increment of spot overlap percentage. However, the value of the percentage of spot overlap decreases with the increase of scanning speed at constant laser spot diameter and pulse frequency. As the scanning speed increases there is less interaction time between the laser beam and the workpiece surface which results in a decrement in spot overlapping percentage. The mathematical relationship of the transverse overlaps and various variables are defined in Eq. (2) [21].

Transverse Overlap Percentage $=\left(1-\frac{D_{T}}{D}\right) \times 100 \%$

The transverse feed is denoted by $D_{T}$ (in $\mathrm{mm}$ ) and the spot diameter of laser is denoted by $D$ (in $\mathrm{mm}$ ). According to Eq. (2), the transverse overlap percentage is inversely proportional to transverse feed. This is due to the fact that

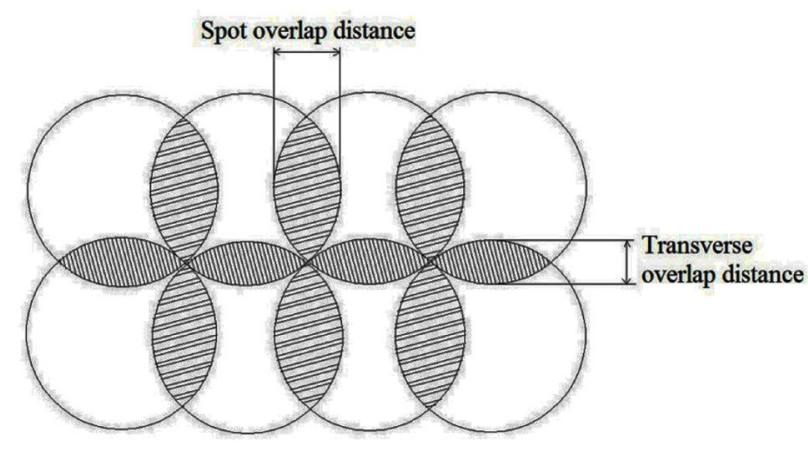

Fig. 4 Details of laser scanning approach showing spot and transverse overlap with the increase of transverse feed, the distance between the two laser beam spots is also increased which results in a reduction in the values of transverse overlapping percentage. During laser micro-channel milling operation of zirconia ceramics, the basic understanding of spots overlaps and transverse overlap of the laser beam is very much needed as the machining surface texture to be generated depends on these overlap factors. The amount or percentage of these two overlaps should be chosen very carefully as these overlaps depend only upon the various parametric combinations' settings of related process parameters. The values of these parameters (spot overlap and transverse overlap) are set more than $75 \%$ which ensures higher removal of material while machining [22].

\subsection{Experimental investigation}

During trial experimentations, it is observed that spattering of laser beam is occurred at some settings of process parameters or in some settings laser beam does not able to penetrate on the workpiece surface. Therefore, it is a challenging task for proper selection of process parameters and their ranges. Because of this reason, extensive tryout experiments are done to select the parameters and their ranges for obtaining micro-channel. The values of some process variables are kept fixed as air pressure at $4 \mathrm{kgf} / \mathrm{cm}^{2}$ and pulse width at $80 \%$ during experimentation. After conducting some trial experimentation, these constant values were chosen. The upper value of air pressure is preferred to ensure the removal of a melted or vaporized portion of work material from the laser-irradiated zone. Experimental strategy (OFAT, one factor at a time) is implemented for the conduct of experiments. Table 3 enlists the various process variables taken into consideration for experimentation.

Measurement (at 5X magnification) of the HAZ width is accomplished with the help of STM7-LM (made by Olympus Inc) optical measuring microscope and at various locations of micro-channel measurement is performed five times then an average of these values was taken for further analysis. Equation (3) is used for the measurement of HAZ width:

Table 3 Details of process parameters and ranges

\begin{tabular}{lc}
\hline Process parameters & Values \\
\hline Average power & $10,11.25,12.50,13.75,15 \mathrm{~W}$ \\
Pulse frequency & $55,60,65,70,75 \mathrm{kHz}$ \\
Scanning speed & $1,5,7,9,11 \mathrm{~mm} / \mathrm{s}$ \\
No of pass & $3,5,7,9,11$ \\
Transverse Feed & $0.001,0.003,0.005,0.007,0.009 \mathrm{~mm}$ \\
\hline
\end{tabular}


$H_{W}=\frac{H_{T W}-K_{W}}{2}$,

where $H_{W}, H_{T W}$ and $K_{W}$ corresponds to HAZ width, HAZ total width and kerf width respectively.

\section{Results and discussion}

\subsection{Effect of process parameters on widths of the Heat}

\section{Affected Zone}

The effect of process variables on HAZ width has been shown in Figs. 5 to 7. Fig. 5 depicts that average power has a considerable impact on HAZ width while keeping other process variables constant such as scanning speed

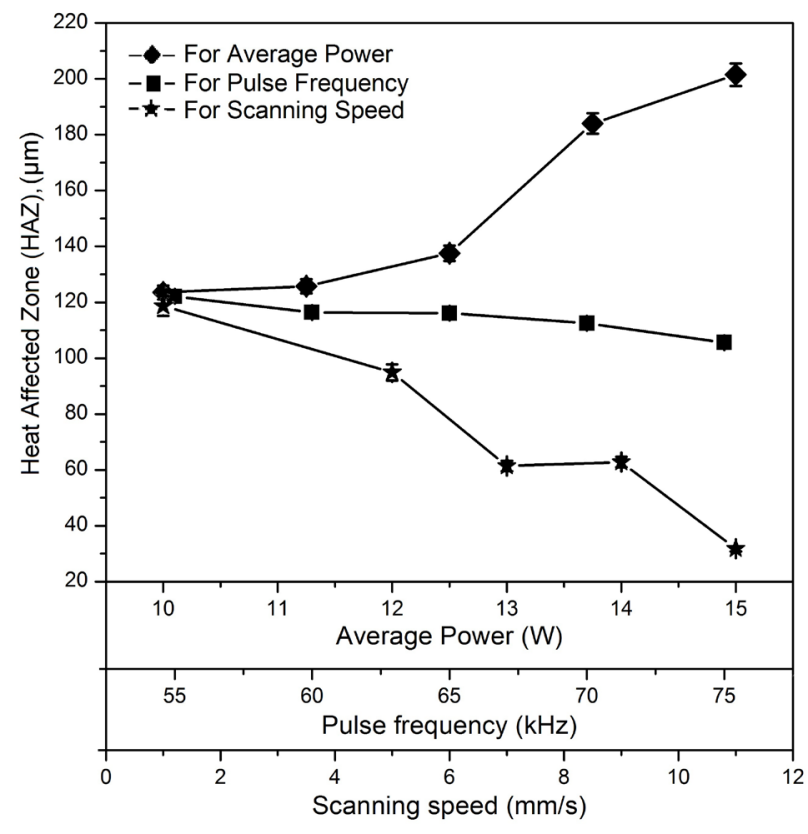

Fig. 5 Effect of average power, pulse frequency and scanning speed on Heat Affected Zone (HAZ) width

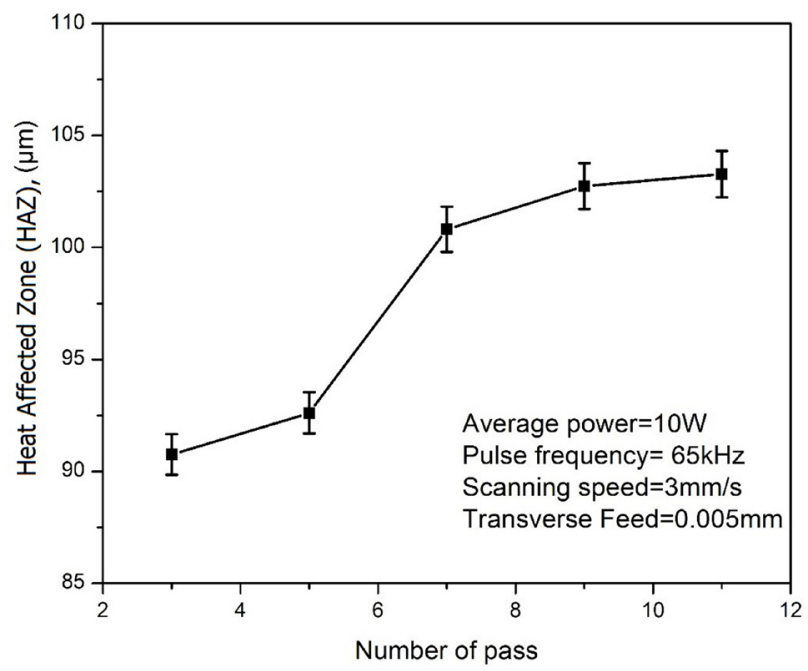

Fig. 6 Effect of number of pass on Heat Affected Zone (HAZ) width

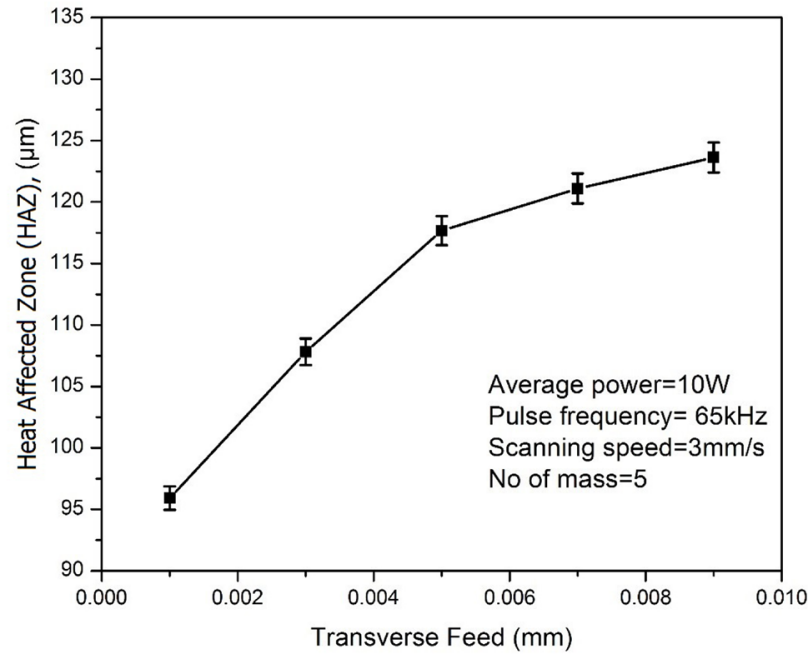

Fig. 7 Effect of transverse feed on Heat Affected Zone (HAZ) width

$(3 \mathrm{~mm} / \mathrm{s})$, pulse frequency $(50 \mathrm{kHz})$, number of pass ( $\mathrm{sin}$ gle), and transverse feed $(0.005 \mathrm{~mm})$. It is perceived from the figure that the HAZ width increases with laser power. According to Eq. (4) [23], the laser beams peak power $\left(P_{\text {peak }}\right)$ is directly related to laser power $\left(P_{\text {avg }}\right)$ while Pulse Frequency (PF) and Pulse Duration (PD) are kept constant.

$P_{\text {avg }}=P_{\text {peak }} \times \mathrm{PF} \times \mathrm{PD}$

Maximum peak power rises the temperature of the impinged machining surface above the melting point which is sufficient to change the phase, and result in material melting or evaporation.

With the increase of average power, the rate of heat generation on the work sample surface is also increased which results in an increment of temperature on the machining zone. The mass flow rate of zirconia ceramic from the surfaces increases with the increment of temperature [24]. Some portion of the material is not melted or evaporated but whose microstructure and properties are changed due to the generation of high heat. Increase in power leads to more dispersion of the heat during the machining accountable for higher values of the width of HAZ. The lower HAZ width is achieved as $123.51 \mu \mathrm{m}$ at a laser power of $10 \mathrm{~W}$. Values of other factors are mentioned above.

It is also observed from Fig. 5 that the effect of pulse frequency on HAZ width while keeping other control variables constant such as scan speed $(3 \mathrm{~mm} / \mathrm{s})$, laser beam average power $(10 \mathrm{~W})$, transverse feed $(0.005 \mathrm{~mm})$, and the number of pass (single). In addition, the width of the HAZ decreases with pulse frequency. The decrement rate is lower with the change in pulse frequency. This parameter defines the number of times the "burst" of photons impinged on the surface per unit duration of a cycle. 
Equation (4) depicts that pulse frequency is inversely proportional to the peak power of the laser. Low frequencies at high peak power will increase the temperature of the surface very quickly which causes material vaporization from the work sample. At higher pulse frequency, the lower peak power will yield lesser energy which results in the decrement of sputtering and re-solidification of the melted portion from the machining zone. However, the pulse frequency is also directly related to the amount of overlap between two successive laser spots (spot overlap percentage) which led to a lesser amount of scattering beside the micro-channel at maximum values of pulse frequency. The lower HAZ width is achieved as $105.57 \mu \mathrm{m}$ at a parametric combination of pulse frequency $(75 \mathrm{kHz})$. The values of other factors are mentioned above.

Fig. 5 also shows that the impact of scan speed on the HAZ width of the micro-channel while keeping other variables constant like pulse frequency $(65 \mathrm{kHz})$, average power $(10 \mathrm{~W})$, transverse feed $(0.005 \mathrm{~mm})$, and the number of pass (single). Fig. 5 also depicts that the HAZ width decreases gradually with the scan speed. The interaction time between laser beam and workpiece surface is related with the scanning speed [3]. Equation (5) describes the relationship between the interaction time ( $T$ ), laser beam spot diameter $(D)$ and scanning speed $(v)$. It is seen from the equation that the interaction time is inversely related with the scanning speed at constant laser beam spot diameter.

$T=\frac{D}{v}$

As the scanning speed increases, the duration between the laser beam and work sample surface is also decreased which is responsible for lower heat input on the cutting zone of the substrate surface. The excess material is carried away from the machining zone using a pressure gas jet and the chances of propagation of heat from the micro-channel also become lower when the value of scanning speed at the higher side. As a result of this, comparatively lower surface damage and the thermally affected zone is achieved. The lower HAZ width is achieved as $31.74 \mu \mathrm{m}$ at $11 \mathrm{~mm} / \mathrm{s}$ value of scanning speed. The values of other factors are mentioned above.

In Fig. 6, the effect of pass number on HAZ width is depicted while keeping other factors such as scanning speed $(3 \mathrm{~mm} / \mathrm{s})$, pulse frequency $(65 \mathrm{kHz})$, transverse feed $(0.005 \mathrm{~mm})$, and average power $(10 \mathrm{~W})$. The graphical plot shows that with the number of pass, the value of HAZ width increases. As the number of pass increases, layer by layer material is removed from the workpiece surface. Thus, laser pulse energy at the machining zone rises quickly with the number of pass. Hence, the workpiece does not get adequate time for removal of the vaporized or melted portion from the surface. The excess material is again solidified at the sidewall of the micro-channel and sputtering of the melted material is occurred at the machining area. It is also witnessed that a thin layer of HAZ is surrounded at the micro-channel edges. The lower HAZ width is achieved as $90.75 \mu \mathrm{m}$ at the number of pass at 3 . The values of other factors are mentioned above.

Fig. 7 shows the effect of transverse feed on HAZ width while keeping other process variables constant such as scanning speed $(3 \mathrm{~mm} / \mathrm{s})$, average power $(10 \mathrm{~W})$, pulse frequency $(65 \mathrm{kHz})$, and the number of pass (5). It is observed that with the rise in transverse feed, the HAZ width increases as the distance between the two laser beam spots is also increased. Transverse overlapping percentage plays a vital role in the generation of micro-channel and it is inversely proportional with transverse feed [21]. Higher values of transverse overlap percentage led to more uniform material removal during machining. However, as the value of transverse feed increases, pulse energy on the work sample become insufficient and the surface does not get significant time duration for the elimination of surplus material. Due to this reason, dispersion of heat occurs from the micro-channel which is responsible for the higher value of HAZ width. The lower HAZ width is achieved as $95.91 \mu \mathrm{m}$ at transverse feed at $0.001 \mathrm{~mm}$. The values of other factors are mentioned above.

Fig. 8 depicts the optical micrographs of the micro-channel showing a re-solidified portion on the micro-channel bottom surface. Micro-channel was machined at a pulse frequency at $65 \mathrm{kHz}$ and average power of $10 \mathrm{~W}$. Microscopic images were also examined for HAZ width analysis.

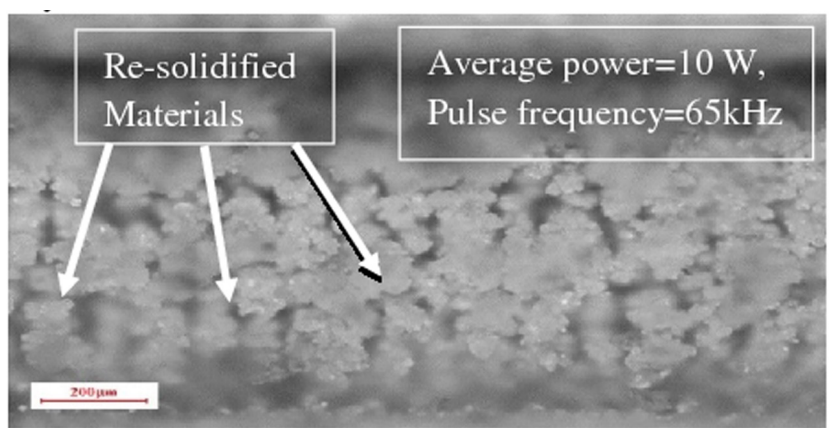

Fig. 8 Optical microscopic images (50X) of micro-channel milling showing the resolidified materials at the bottom of channel 
Fig. 9 shows the microscopic image of the HAZ width at the parametric combination. From the various microscopic images, it can be stated that there is a huge impact of process variables on the width of the Heat Affected Zone created at micro-channel. Comparing Fig. 9 (a) and (b) when laser average power is increased from 12.5 to $13.75 \mathrm{~W}$, the HAZ width increased from 137.54 to $184 \mu \mathrm{m}$ and this is mainly due to more power density at the micro-channel zone. Comparing Fig. 9 (c) and (d) when scanning speed is increased from 5 to $11 \mathrm{~mm} / \mathrm{s}$, the HAZ width is reduced from 94.88 to $31.74 \mu \mathrm{m}$. This is mainly due to less duration of laser-matter interaction at higher scanning speed and thus, material from the micro-channel is removed quickly.

\section{Conclusions}

The width of the HAZ obtained during the micro-channel milling of zirconia by using $50 \mathrm{~W}$ ytterbium-doped fiber laser machining is investigated. The effect process parameters on HAZ width have been deliberate by using various graphical plots as well as optical microscopic images. From the experimentation and results, the following conclusions are listed below:

1. It is found that laser beam average power has a great effect on HAZ width. With the laser power increase, the HAZ width rises. Higher peak power leads to more dispersion of the heat are occurred during the machining which is responsible for higher values of the width of HAZ.

2. With a rise in pulse frequency, the values of HAZ width decreases. At higher pulse frequency, the lower peak power will produce less energy which results in the decrement of sputtering and re-solidification of the melted portion from the machining area.

3. It is also witnessed that HAZ width reduces gradually with an increment of scan speed. As the value of scan speed increases, the interaction time decreases between the laser beam and work sample responsible for lower heat input on the cutting zone of the substrate surface.

\section{References}

[1] Meijer, J. "Laser beam machining (LBM), state of the art and new opportunities", Journal of Materials Processing Technology, 149(1-3), pp. 2-17, 2004.

https://doi.org/10.1016/j.jmatprotec.2004.02.003

[2] Dubey, A. K., Yadava, V. "Laser beam machining-A review", International Journal of Machine Tools and Manufacture, 48(6), pp. 609-628, 2008 . https://doi.org/10.1016/j.ijmachtools.2007.10.017

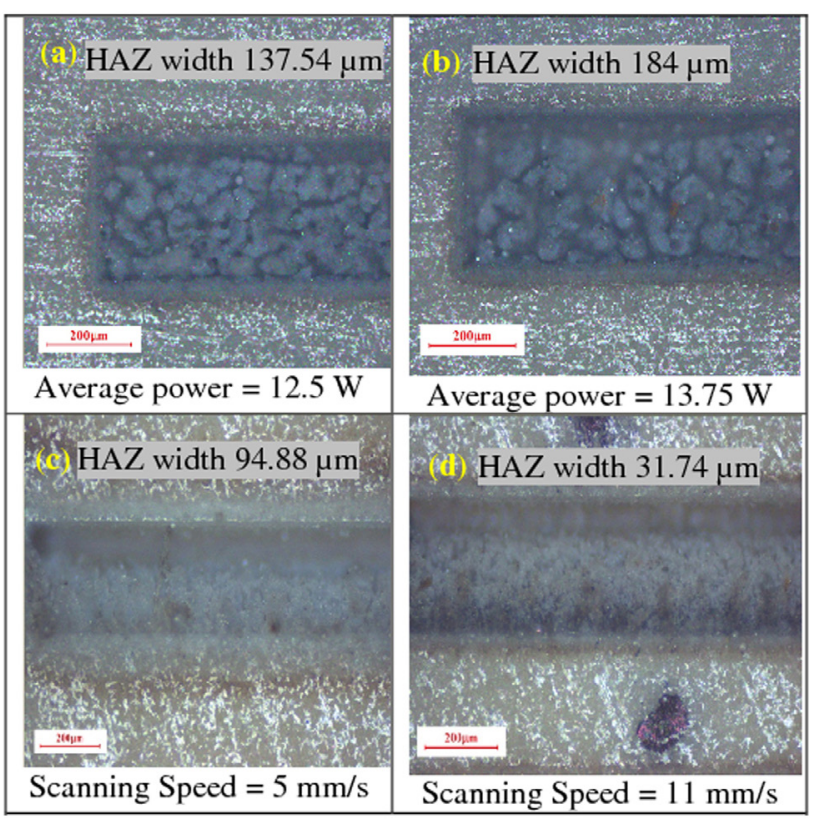

Fig. 9 Optical microscopic images of micro-channel milling machined at various parametric conditions; (a) and (b) Pulse frequency $=50 \mathrm{kHz}$,

Scanning Speed $=3 \mathrm{~mm} / \mathrm{s}$, Transverse Feed $=0.005 \mathrm{~mm}$; (c) and

(d) Average power $=10 \mathrm{~W}$, Pulse frequency $=65 \mathrm{kHz}$, Transverse Feed $=0.005 \mathrm{~mm}$

4. With a rise in the number of pass, the HAZ width increases. Laser pulse energy at the machining zone increases rapidly with the rise in the number of pass which results in solidification of the excess material is again solidified at the sidewall of the micro-channel and sputtering of the melted material is occurred at the machining zone.

5. It can be seen that with the rise in transverse feed, the HAZ width increases. As the value of transverse feed increases, pulse energy on the work sample becomes insufficient and the surface does not get effective time duration for the elimination of surplus material.

The present research may be used for future research work associated with HAZ width of micro-channel milling of various HSTR material and also for investigating other process variables to obtain improved features of micro-channel.

[3] Saklakoglu, I. E., Kasman, S. "Investigation of micro-milling process parameters for surface roughness and milling depth", The International Journal of Advanced Manufacturing Technology, 54(5), pp. 567-578, 2011.

https://doi.org/10.1007/s00170-010-2953-3

[4] Sahu, A. K., Jha, S. "Microchannel fabrication and metallurgical characterization on titanium by nanosecond fiber laser micromilling", Materials and Manufacturing Processes, 35(3), pp. 279-290, 2020. https://doi.org/10.1080/10426914.2020.1718702 
[5] Samant, A. N., Dahotre, N. B. "Laser machining of structural ceramics-A review", Journal of the European Ceramic Society, 29(6), pp. 969-993, 2009.

https://doi.org/10.1016/j.jeurceramsoc.2008.11.010

[6] Pham, D. T., Dimov, S. S., Petkov, P. V. "Laser milling of ceramic components", International Journal of Machine Tools and Manufacture, 47(3-4), pp. 618-626, 2007. https://doi.org/10.1016/j.ijmachtools.2006.05.002

[7] Bona, A. D., Pecho, O. E., Alessandretti, R. "Zirconia as a Dental Biomaterial", Materials, 8(8), pp. 4978-4991, 2015.

https://doi.org/10.3390/ma8084978

[8] Leone, C., Papa, I., Tagliaferri, F., Lopresto, V. "Investigation of CFRP laser milling using a $30 \mathrm{~W}$ Q-switched Yb:YAG fiber laser: Effect of process parameters on removal mechanisms and HAZ formation", Composites Part A: Applied Science and Manufacturing, 55, pp. 129-142, 2013.

https://doi.org/10.1016/j.compositesa.2013.08.004

[9] Zhao, K., Jia, Z., Liu, W., Ma, J., Wang, L. "Material removal with constant depth in $\mathrm{CNC}$ laser milling based on adaptive control of laser fluence", The International Journal of Advanced Manufacturing Technology, 77(5), pp. 797-806, 2015.

https://doi.org/10.1007/s00170-014-6481-4

[10] Darwish, S., Ahmed, N., Alahmari, A. M., Mufti, N. A. "A study of micro-channel size and spatter dispersion for laser beam micro-milling", Materials and Manufacturing Processes, 32(2), pp. 171-184, 2017.

https://doi.org/10.1080/10426914.2016.1176188

[11] Wang, X., Huang, Y., Xing, Y., Fu, X., Zhang, Z., Ma, C. "Fabrication of micro-channels on $\mathrm{Al}_{2} \mathrm{O}_{3} / \mathrm{TiC}$ ceramics using picosecond laser induced plasma micromachining", Journal of Manufacturing Processes, 44, pp. 102-112, 2019.

https://doi.org/10.1016/j.jmapro.2019.05.048

[12] Liu, Y., Liu, L., Deng, J., Meng, R., Zou, X., Wu, F. "Fabrication of micro-scale textured grooves on green $\mathrm{ZrO}_{2}$ ceramics by pulsed laser ablation", Ceramics International, 43(8), pp. 6519-6531, 2017. https://doi.org/10.1016/j.ceramint.2017.02.074

[13] Parry, J. P., Shephard J. D., Dear, F. C., Jones, N., Weston, N., Hand, D. P. "Nanosecond-Laser Postprocessing of MillisecondLaser-Machined Zirconia (Y-TZP) surfaces", International Journal of Applied Ceramic Technology, 5(3), pp. 249-257, 2008. https://doi.org/10.1111/j.1744-7402.2008.02222.x

[14] Abdo, B. M. A., Ahmed, N., El-Tamimi, A. M., Anwar, S., Alkhalefah, H., Nasr, E. A. "Laser beam machining of zirconia ceramic: An investigation of micro-machining geometry and surface roughness", Journal of Mechanical Science and Technology, 33(4), pp. 1817-1831, 2019. https://doi.org/10.1007/s12206-019-0334-x
[15] Dear, F. C., Shephard, J. D., Wang, X., Jones, J. D. C., Hand, D. P. "Pulsed Laser Micromachining of Yttria-Stabilized Zirconia Dental Ceramic for Manufacturing", International Journal of Applied Ceramic Technology, 5(2), pp. 188-197, 2008. https://doi.org/10.1111/j.1744-7402.2008.02203.x

[16] Snakenborg, D., Klank, H., Kutter, J. P. "Microstructure fabrication with a $\mathrm{CO}_{2}$ laser system", Journal of Micromechanics and Microengineering, 14(2), pp. 182-189, 2003. https://doi.org/10.1088/0960-1317/14/2/003

[17] Deng, D., Wan, W., Huang, Q., Huang, X., Zhou, W. "Investigations on laser micromilling of circular micro pin fins for heat sink cooling systems", The International Journal of Advanced Manufacturing Technology, 87(1), pp. 151-164, 2016. https://doi.org/10.1007/s00170-016-8468-9

[18] Singh, R., Alberts, M. J., Melkote, S. N. "Characterization and prediction of the heat-affected zone in a laser-assisted mechanical micromachining process", International Journal of Machine Tools and Manufacture, 48(9), pp. 994-1004, 2008.

https://doi.org/10.1016/j.ijmachtools.2008.01.004

[19] Pan, Z., Feng, Y., Hung, T. P., Jiang, Y. C., Hsu, F. C., Wu, L. T., Lin, C. F., Lu, Y. C., Liang, S. Y. "Heat affected zone in the laser-assisted milling of Inconel 718", Journal of Manufacturing Processes, 30, pp. 141-147, 2017. https://doi.org/10.1016/j.jmapro.2017.09.021

[20] Thawari, G., Sundar, J. K. S., Sundararajan, G., Joshi, S. V. "Influence of process parameters during pulsed Nd:YAG laser cutting of nickel-base superalloys", Journal of Materials Processing Technology, 170(1-2), pp. 229-239, 2005. https://doi.org/10.1016/j.jmatprotec.2005.05.021

[21] Vora, H. D., Dahotre, N. B. "Surface topography in three-dimensional laser machining of structural alumina", Journal of Manufacturing Processes, 19, pp. 49-58, 2015. https://doi.org/10.1016/j.jmapro.2015.04.002

[22] Kibria, G., Doloi, B., Bhattacharyya, B. "Optimisation of Nd:YAG laser micro-turning process using response surface methodology", International Journal of Precision Technology (IJPTECH), 3(1), pp. 14-36, 2012.

https://doi.org/10.1504/IJPTECH.2012.045986

[23] Kibria, G., Doloi, B., Bhattacharyya, B. "Investigation into the effect of overlap factors and process parameters on surface roughness and machined depth during micro-turning process with Nd:YAG laser", Optics \& Laser Technology, 60, pp. 90-98, 2014. https://doi.org/10.1016/j.optlastec.2014.01.009

[24] Shukla, P. P., Lawrence, J. "Distribution of temperature during fibre laser radiation and effects thereon phase transformation of $\mathrm{ZrO}_{2}$ engineering ceramic", Surface Engineering, 27(10), pp. 742-748, 2011. https://doi.org/10.1179/1743294411Y.0000000002 